\title{
Erratum: Simulation of single-qubit open quantum systems [Phys. Rev. A 90, 022331 (2014)]
}

\author{
Ryan Sweke, Ilya Sinayskiy, and Francesco Petruccione
}

(Received 28 May 2017; published 14 June 2017)

DOI: 10.1103/PhysRevA.95.069905

In Sec. IV it was claimed that $T_{t}$ could be approximated via a finite sequence of quantum channels $T_{t^{\prime}}^{(j)}$ through the use of higher-order Suzuki-Lie-Trotter (SLT) integrators of the form $\left[S_{2 k}(t / r)\right]^{r L_{1}}$ for arbitrary $k \in \mathbb{N}$. However, for $k>1$ it is in fact the case that $\left(1-4 p_{k}\right)<0$, and therefore applying the recursion rule in Eq. (29) allows us to see that for all $k>1$ implementation of $S_{2 k}(\lambda)$ requires the simulation of multiple propagators $T_{\tilde{t}}^{(j)}$ with $\tilde{t}<0$ [1]. As such propagators are not quantum channels (in particular they may violate complete positivity, or even positivity) [2], it is in fact only possible to exploit first-order integrators $(k=1)$, and as such, Theorem 2 is only valid for $k=1$ as opposed to all $k \in \mathbb{N}$. In light of these considerations, Theorem 2 should be replaced with the following theorem, which is an optimized version of Theorem 2 when restricted to the case of $k=1$.

Theorem 1. Let $1 \geqslant \epsilon>0$ be such that $(9 / 2) L_{2} m t \geqslant \epsilon$, then for

$$
r \geqslant \frac{\sqrt{2 L_{2}}(m t)^{3 / 2}}{\epsilon^{1 / 2}},
$$

we have that

$$
\left\|\exp \left(t \sum_{j=1}^{m} \mathcal{L}_{j}\right)-\left[S_{2}\left(\hat{\mathcal{L}}_{1}, \ldots, \hat{\mathcal{L}}_{m}, t / r\right)\right]^{r L_{1}}\right\|_{1 \rightarrow 1} \leqslant \epsilon,
$$

and the number of exponentials required is bounded by

$$
N_{\exp } \leqslant(2 m-1) \frac{\sqrt{2 L_{2}} L_{1}(m t)^{3 / 2}}{\epsilon^{1 / 2}} .
$$

The original proof of Theorem 2 should be replaced with the analogous proof for the above Theorem 1, which is as follows: First, we replace Lemma 1 with the following lemma, which can be proven using the exact same proof as described in Ref. [3], provided one restricts oneself to $k=1$, replaces the 1 norm with the $(1 \rightarrow 1)$ norm and notes that $\|T\|_{1 \rightarrow 1}=1$ for any quantum channel $T$ [4].

Lemma 1. For $(2 / 3) m|\lambda| \leqslant 1$ and

$$
\left\|\hat{\mathcal{L}}_{m}\right\|_{1 \rightarrow 1} \leqslant \cdots \leqslant\left\|\hat{\mathcal{L}}_{2}\right\|_{1 \rightarrow 1} \leqslant\left\|\hat{\mathcal{L}}_{1}\right\|_{1 \rightarrow 1}=1
$$

we have that

$$
\left\|\exp \left(\lambda \sum_{j=1}^{m} \hat{\mathcal{L}}_{j}\right)-S_{2}(\lambda)\right\|_{1 \rightarrow 1} \leqslant 2\left\|\hat{\mathcal{L}}_{2}\right\|_{1 \rightarrow 1}(m|\lambda|)^{3},
$$

where $S_{2}(\lambda)=S_{2}\left(\hat{\mathcal{L}}_{1}, \ldots, \hat{\mathcal{L}}_{m}, \lambda\right)$.

We can then utilize the modified Lemma 1 above and Lemma 2 to obtain

$$
\left\|\exp \left(t \sum_{j=1}^{m} \mathcal{L}_{j}\right)-\left[S_{2}\left(\frac{t}{r}\right)\right]^{r L_{1}}\right\|_{1 \rightarrow 1} \leqslant \frac{2 L_{2}(m t)^{3}}{r^{2}}
$$

Therefore, taking

$$
r \geqslant \frac{\sqrt{2 L_{2}}(m t)^{3 / 2}}{\epsilon^{1 / 2}},
$$

and enforcing that $(9 / 2) L_{2} m t \geqslant \epsilon$ ensures that both the conditions of Lemma 1 and the bound (2) are satisfied. Finally, from Eq. (31) one then can see that the total number of exponentials required is indeed as stated in Eq. (3).

In light of this necessary restriction to the case of $k=1$, the complete algorithm presented in Sec. VI should be modified as follows:

(1) Given $\mathcal{L}$, obtain as per Sec. III the spectral decomposition,

$$
\mathcal{L}=\sum_{k=0}^{4} \lambda_{k} \mathcal{L}_{k}
$$


as well as $G_{k}$ and $\theta_{k}$ specifying the decomposition,

$$
A_{k}=G_{k} A_{\left(\theta_{k}\right)} G_{k}^{T}
$$

for all $k \in[1,3]$.

(2) Choose the desired approximation accuracy $\epsilon$ as well as the simulation time $t$. Using Eq. (28) construct $S_{2}(t / r)$ with

$$
r=\frac{\sqrt{2 L_{2}}(m t)^{3 / 2}}{\epsilon^{1 / 2}} .
$$

(3) Implement $S_{2}(t / r) L_{1} r$ times using

$$
T_{t^{\prime}}^{(k)}(\rho)=U_{k}^{\dagger}\left[T_{t^{\prime}}^{\left(\theta_{k}\right)}\left(U_{k} \rho U_{k}^{\dagger}\right)\right] U_{k},
$$

where $\lambda_{k}, L_{1}$, and $r$ have been incorporated into $t^{\prime}, U_{k}$ is obtained from $G_{k}$ as per Sec. III and $T_{t^{\prime}}^{\left(\theta_{k}\right)}$ is implemented via classical random sampling of the circuits derived in Sec. V.

This algorithm now requires $O\left[\left(\|\mathcal{L}\|_{1 \rightarrow 1} t\right)^{3 / 2} / \epsilon^{1 / 2}\right]$ single-qubit and controlled-NOT (CNOT) gates as opposed to $O\left[\left(\|\mathcal{L}\|_{1 \rightarrow 1} t\right)^{1+1 / 2 k} / \epsilon^{1 / 2 k}\right]$ single-qubit and CNOT gates as originally claimed.

In addition, the following typographical errors should be corrected. Equation (55) for $m_{3}$ should read $m_{3}=\sin \left(2 \theta_{k}\right)\left(\Lambda_{3}-1\right)$ as opposed to $m_{3}=\sin \left(2 \theta_{k}\right)\left(1-\Lambda_{3}\right)$. Equations (72) and (73) for $\phi_{1}$ and $\phi_{2}$ should be replaced with

$$
\begin{aligned}
& \phi_{1}=\arccos \left(\frac{\Lambda_{1}+\Lambda_{2}}{a d}\right), \\
& \phi_{2}=\arccos \left(\frac{\Lambda_{1}-\Lambda_{2}}{b c}\right),
\end{aligned}
$$

and finally Eqs. (76) and (77) should be replaced with

$$
K_{1}^{1}=\frac{1}{\sqrt{2}}\left(\begin{array}{cc}
0 & c \\
b e^{i \phi_{2}} & 0
\end{array}\right), \quad K_{2}^{1}=\frac{1}{\sqrt{2}}\left(\begin{array}{cc}
a e^{-i \phi_{1}} & 0 \\
0 & d
\end{array}\right)
$$

and

$$
K_{1}^{2}=\frac{1}{\sqrt{2}}\left(\begin{array}{cc}
0 & c \\
b e^{-i \phi_{2}} & 0
\end{array}\right), \quad K_{2}^{2}=\frac{1}{\sqrt{2}}\left(\begin{array}{cc}
a e^{i \phi_{1}} & 0 \\
0 & d
\end{array}\right) .
$$

The authors would like to thank $\mathrm{T}$. Li and A. Childs for bringing the issues concerning higher-order SLT integrators to our attention and Z. Tian for pointing out the typographical errors in Eqs. (55), (72), (73), (76), and (77).

[1] M. Suzuki, Phys. Lett. A 146, 319 (1990).

[2] H. P. Breuer and F. Petruccione, The Theory of Open Quantum Systems (Oxford University Press, New York, 2002).
[3] A. Papageorgiou and C. Zhang, Quant. Inf. Process. 11, 541 (2012).

[4] J. Watrous, Quant. Inf. Comput. 5(1), 58 (2005). 